\title{
Correction to: Review of GPS collar deployments and performance on nonhuman primates
}

\author{
Kerry M. Dore ${ }^{1}(1) \cdot$ Malene F. Hansen ${ }^{2,3} \cdot$ Amy R. Klegarth $^{4} \cdot$ Claudia Fichtel $^{5} \cdot$ Flávia Koch $^{5} \cdot$ Andrea Springer $^{5}$. \\ Peter Kappeler ${ }^{5}$. Joyce A. Parga ${ }^{6}$. Tatyana Humle ${ }^{7}$. Christelle Colin ${ }^{7}$. Estelle Raballand ${ }^{7}$. Zhi-Pang Huang ${ }^{8}$. \\ Xiao-Guang $\mathrm{Qi}^{9,10}$. Anthony Di Fiore ${ }^{11}$. Andrés Link ${ }^{12}$. Pablo R. Stevenson ${ }^{12}$. Danica J. Stark ${ }^{13,14}$. Noeleen Tan ${ }^{15}$. \\ Christa A. Gallagher ${ }^{16}$. C. Jane Anderson ${ }^{17}$. Christina J. Campbell ${ }^{18} \cdot$ Marina Kenyon $^{19} \cdot$ Paula Pebsworth $^{1,20}$. \\ David Sprague $^{21} \cdot$ Lisa Jones-Engel ${ }^{4} \cdot$ Agustín Fuentes $^{22}$
}

Published online: 5 February 2020

(c) Japan Monkey Centre and Springer Japan KK, part of Springer Nature 2020

\section{Correction to: Primates} https://doi.org/10.1007/s10329-020-00793-7

In the original publication of the article, figure 1 was wrongly published as a duplication of figure 2 . The correct Fig. 1 is given in this correction.

The original article can be found online at https://doi.org/10.1007/ s10329-020-00793-7.

Kerry M. Dore

kerrymdore@gmail.com

Malene F. Hansen

malenefriishansen@gmail.com

Amy R. Klegarth

amy.klegarth@gmail.com

Agustín Fuentes

afuentes@nd.edu

1 Department of Anthropology, Baylor University, One Bear Place, Waco, TX 76798, USA

2 Research and Conservation, Copenhagen Zoo, 2000 Frederiksberg C, Denmark

3 Animal Behaviour Group. Section for Ecology and Evolution, University of Copenhagen, 2100 Copenhagen, Denmark

4 Department of Anthropology, University of Washington, 230 Raitt Hall, Seattle, WA 98105, USA

5 Behavioral Ecology and Sociobiology Unit, German Primate Center, 37077 Göttingen, Germany
6 Department of Anthropology, California State University, Los Angeles, Los Angeles, CA 90032, USA

7 Durrell Institute of Conservation and Ecology, School of Anthropology and Conservation, University of Kent, Canterbury CT2 7NR, UK

8 Institute of Eastern-Himalaya Biodiversity Research, Dali University, Dali 671003, Yunnan, China

9 College of Life Sciences, Northwest University, Xian 710069, Shanxi, China

10 Shaanxi Key Laboratory for Animal Conservation, Northwest University, Xian 710069, Shaanxi, China

11 Department of Anthropology, University of Texas Austin, Austin, TX 78712, USA

12 Department of Biological Science, University of Los Andes, Bogota, Colombia

13 Danau Girang Field Centre, c/o Sabah Wildlife Department, 88100 Kota Kinabalu, Sabah, Malaysia

14 Organisms and Environment Division, Cardiff School of Biosciences, Cardiff University, Cardiff CF10 3AX, UK 


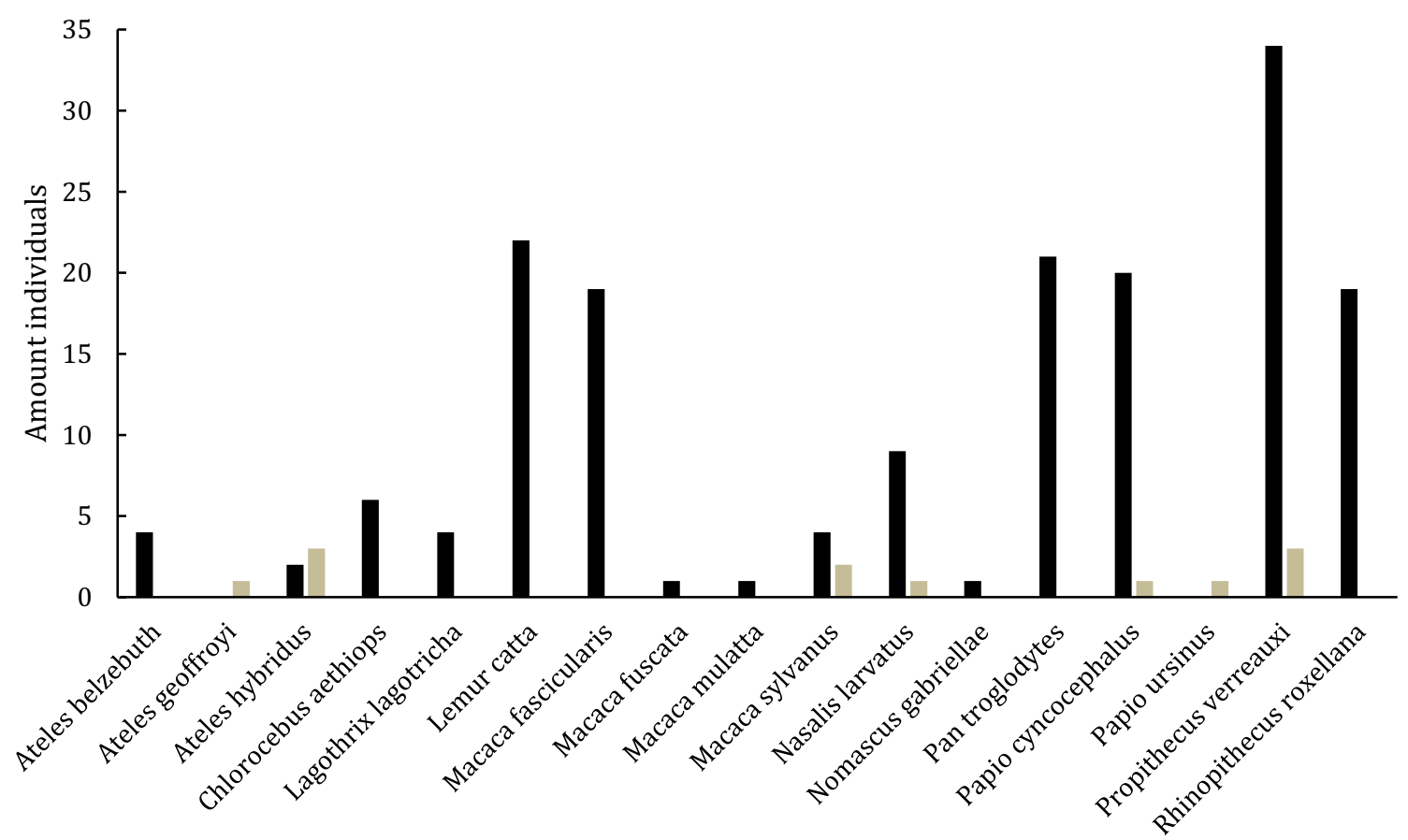

Primate species

- Adult $\square$ Subadult

Fig. 1 Number of GPS collar deployments by species and age group

15 Singapore National Parks Board, Singapore, Singapore

16 Department of Biomedical Science, Center for Conservation Medicine and Ecosystem Health, Ross University School of Veterinary Medicine, West Indies, Saint Kitts and Nevis

17 Department of Wildlife Ecology and Conservation, University of Florida, Gainesville, FL 32611, USA

18 Department of Anthropology, California State University Northridge, Northridge, CA 91330, USA

19 Dao Tien Endangered Primate Species Centre, Tan Phu, Dong Nai Province, Vietnam

20 National Institute of Advanced Studies, Indian Institute of Science Campus, Bangalore, India

21 National Agriculture and Food Research Organization, 3-1-3 Kannondai, Tsukuba 305-8604, Japan

22 Department of Anthropology, University of Notre Dame, 648 Flanner Hall, Notre Dame, IN 46656, USA
Publisher's Note Springer Nature remains neutral with regard tojurisdictional claims in published maps and institutional affiliations. 